TRANSACTIONS OF THE

AMERICAN MATHEMATICAL SOCIETY

Volume 358, Number 7, Pages 2837-2853

S 0002-9947(06)03938-9

Article electronically published on February 6, 2006

\title{
UNIQUE CONTINUATION FOR THE TWO-DIMENSIONAL ANISOTROPIC ELASTICITY SYSTEM AND ITS APPLICATIONS TO INVERSE PROBLEMS
}

\author{
GEN NAKAMURA AND JENN-NAN WANG
}

\begin{abstract}
Under some generic assumptions we prove the unique continuation property for the two-dimensional inhomogeneous anisotropic elasticity system. Having established the unique continuation property, we then investigate the inverse problem of reconstructing the inclusion or cavity embedded in a plane elastic body with inhomogeneous anisotropic medium by infinitely many localized boundary measurements.
\end{abstract}

\section{INTRODUCTION}

Assume that $\mathcal{B}$ is an anisotropic elastic body and the reference configuration of $\mathcal{B}$ is $\Omega$, a bounded open connected set in $\mathbb{R}^{2}$. Let $C(x)=\left(C_{i j k l}(x)\right)$ be the elastic tensor. Here and below, all Latin indices are set to be from 1 to 2 . We assume that the elastic tensor $C$ satisfies the full symmetric properties

$$
C_{i j k l}=C_{j i k l}=C_{k l i j} \quad \forall i, j, k, l .
$$

The displacement equation of equilibrium when there is no body force is given by

$$
\mathcal{L}_{C} u=\nabla \cdot(C(x) \nabla u)=0 \text { in } \Omega,
$$

where $(\nabla u)_{k l}=\partial_{l} u_{k}$ and $(\nabla \cdot G)_{i}=\sum_{j} \partial_{j} g_{i j}$ for any matrix function $G=\left(g_{i j}\right)$. Also, we have used the convention notation

$$
(C H)_{i j}=\sum_{k l} C_{i j k l} h_{k l},
$$

where $H=\left(h_{i j}\right)$ is a $2 \times 2$ matrix. Here $u={ }^{t}\left[u_{1}, u_{2}\right]$ is the displacement vector. In this paper we are concerned with the unique continuation property (UCP) for (1.2). Precisely, we want to know whether $u$ vanishes identically in $\Omega$ whenever it vanishes in some nonempty open subset of $\Omega$.

The unique continuation property for differential equations has a long history. There exists a vast number of literature in this field, especially for scalar differential equations. We will not try to give a full account of its development here. Instead, we only mention some related results on the elasticity system. When the medium is isotropic, the UCP has been established in [1, 4] and 21. Moreover, a strong

Received by the editors January 5, 2004.

2000 Mathematics Subject Classification. Primary 35B60, 74B05; Secondary 74 G75.

Key words and phrases. Unique continuation, anisotropic elasticity system, inverse problems.

The first author was partially supported by Grant-in-Aid for Scientific Research (B)(2)

(No.14340038) of the Japan Society for the Promotion of Science.

The second author was partially supported by the National Science Council of Taiwan.

(C)2006 American Mathematical Society Reverts to public domain 28 years from publication 
unique continuation property for the isotropic system was recently proven in 2] and in [15]. Unlike the isotropic case, the unique continuation property for the inhomogeneous anisotropic elasticity has not been fully explored. In this direction, the authors of this paper have proved the UCP for an elasticity system with residual stress [18]. It is known that this system is no longer isotropic due to the existence of residual stress. A strong unique continuation for the elasticity system with residual stress was recently proved in 14 .

In this paper we want to investigate the UCP for (1.2) when the elastic tensor is anisotropic with finite smoothness. To the authors' knowledge, this problem has not been studied before. For our UCP result in this paper, we assume that the elastic tensor is merely a locally Lipschitz function in $\Omega$. One of the key ideas in our proof is that under suitable conditions the original elasticity system (1.2) can be transformed into a first order elliptic system locally (see (2.11)). Therefore, proving the UCP for (1.2) is now reduced to proving the UCP for (2.11). There were several results on the UCP for first order elliptic systems; see, for example, [3], [5] and [8]. However, in those papers, the matrix function $N$ in (2.11) is assumed to be either (locally) diagonalizable ([3], [5]) by a $C^{1}$ invertible matrix or normal (8]). In this work we are not assuming that $N$ is normal. Instead, we will suppose that near every point $x_{0} \in \Omega$ the quadratic pencil $\Lambda_{11} p^{2}+\Lambda_{12} p+\Lambda_{22}$ (see (2.12) ) has at least one eigenvalue $\theta(x)$ with associated eigenvector $z(x)$, and both are Lipschitz, such that the matrix function $[z, \bar{z}]$ is nonsingular. Under this assumption, we can show that the diagonal blocks of $N$ are Lipschitz diagonalizable. From now on, we say that a matrix function is Lipschitz diagonalizable if it can be similarly transformed to a diagonal matrix with Lipschitz entries by a Lipschitz invertible matrix. Nevertheless, it should be emphasized that the whole matrix $N$ is not necessarily Lipschitz diagonalizable. There are two obstructions preventing it from having this property. On one hand, the two diagonal blocks may have common eigenvalues. On the other hand, the lower left block of $N$, i.e. the $(2,1)$ block of $N$, is only $L^{\infty}$. In view of these two points, it is, in general, not possible to find a Lipschitz invertible matrix to diagonalize $N$. In other words, we have to treat a lower triangular matrix function. Our proof of the UCP for (1.2) via (2.11) relies on some delicate Carleman estimates. To deal with the lower triangular matrix function $N$, we will borrow some ideas from [21] (or [18]). The proof of the UCP is given in Section 2.

Also in Section 2, we give an example to show that the assumption on one eigenpair of the quadratic pencil $\Lambda_{11} p^{2}+\Lambda_{12} p+\Lambda_{22}$ is not too restrictive. This condition is in fact generic. Moreover, it is easy to verify that this assumption holds true when the elastic tensor is isotropic. Therefore, as a by-product of our UCP result, we prove that the UCP holds for the two-dimensional isotropic elasticity system with locally Lipschitz Lamé coefficients, which is an improvement of previous UCP results for the same system in two dimensions. We remark that for the UCP result obtained in 1] or 21, the Lamé coefficients are required to be at least $C^{2}$, while for the strong UCP proved in [2, one needs $C^{1,1}$ coefficients.

Our study of the UCP for the inhomogeneous two-dimensional anisotropic elasticity system (1.2) is motivated by its application to inverse problems. Having proved the UCP for (1.2), in Section 3 we will establish reconstruction algorithms for identifying the inclusion or cavity embedded inside a plane anisotropic elastic body by infinitely many localized boundary measurements with the help of Runge's 
approximation property. For the inclusion identification in the conductive medium, we refer to the pioneer work by Isakov [13. It is well known that Runge's approximation property is an easy consequence of the UCP. Here the reconstruction algorithms of determining the inclusion or cavity are based the probe method developed by Ikehata in [9] (see [10] and references therein for related results). It should be noted that Runge's approximation theorem for the anisotropic elasticity was proved in [1] and 12]. However, the elasticity tensor there was assumed to be either homogeneous or real-analytic. The UCP is an obvious fact in these two situations. For other interesting inverse boundary value problems, we refer readers to a nice survey article [20].

\section{UNIQUE CONTINUATION PROPERTY}

This section is devoted to the proof of the UCP for (1.2) when the elastic tensor $C(x)$ is locally Lipschitz. We will first transform the system (1.2) into a first order elliptic system with appropriate matrix coefficients. Next we will derive some useful Carleman estimates. The proof of the UCP for (1.2) is carried out with the help of those Carleman estimates. Throughout this section, in addition to the symmetric properties (1.1), we assume that the elastic tensor satisfies the strong ellipticity condition, i.e. there exists $\delta>0$ so that for any vectors $a={ }^{t}\left[a_{1}, a_{2}\right]$ and $b={ }^{t}\left[b_{1}, b_{2}\right]$ we have that

$$
C_{i j k l}(x) a_{i} b_{j} a_{k} b_{l} \geq \delta|a|^{2}|b|^{2}
$$

for all $x \in \Omega$.

2.1. First order elliptic system. Our goal here is to transform (1.2) into a first order elliptic system. To this end, we would like to express (1.2) in a more detailed form, namely,

$$
\mathcal{L}_{C} u=\Lambda_{11} \partial_{1}^{2} u+\Lambda_{12} \partial_{1} \partial_{2} u+\Lambda_{22} \partial_{2}^{2} u+R(u)=0 \quad \text { a.e. in } \Omega,
$$

where

$$
\Lambda_{11}=\left(C_{i 1 k 1}\right), \Lambda_{22}=\left(C_{i 2 k 2}\right), \Lambda_{12}=\Phi+{ }^{t} \Phi \text { with } \Phi=\left(C_{i 2 k 1}\right)
$$

and

$$
R(u)=\sum_{j k l}\left(\partial_{j} C_{i j k l}\right) \partial_{l} u_{k}
$$

Since $C(x)$ is locally Lipschitz, it is well known that $\partial_{j} C_{i j k l}(x)$ is locally bounded for all $i, j, k, l$. In other words, $R$ is a first order differential operator with locally bounded coefficients. From the strong ellipticity condition (2.1), we can see that $\Lambda_{11}$ and $\Lambda_{22}$ are invertible (in fact, positive definite). Let $x_{0} \in \Omega$ and $\omega_{x_{0}} \subset \Omega$ be a small open neighborhood of $x_{0}$. We now define $W={ }^{t}\left[w_{1}, w_{2}\right]={ }^{t}\left[u, \partial_{1} u+T \partial_{2} u\right]$ for $x \in \omega_{x_{0}}$, where $T=T(x)$ is a Lipschitz invertible matrix (in $\omega_{x_{0}}$ ) which will be chosen later. Thus, we can compute that

$$
\left\{\begin{array}{l}
\partial_{1} w_{1}=\partial_{1} u=\left(\partial_{1} u+T \partial_{2} u\right)-T \partial_{2} u=-T \partial_{2} w_{1}+w_{2} \\
\partial_{1} w_{2}=\partial_{1}^{2} u+T \partial_{1} \partial_{2} u+\partial_{1} T \partial_{2} w_{1}
\end{array}\right.
$$

Here and below, to simplify expressions, all equations are interpreted in the sense of a.e. in $\omega_{x_{0}}$ unless otherwise indicated. It follows from (2.2) that

$$
\partial_{1}^{2} u=-\Lambda_{11}^{-1}\left(\Lambda_{12} \partial_{1} \partial_{2} u+\Lambda_{22} \partial_{2}^{2} u+R(u)\right)
$$


Using the definition of $w_{2}$, we immediately see that

$$
\partial_{2} w_{2}=\partial_{1} \partial_{2} u+T \partial_{2}^{2} u+\partial_{2} T \partial_{2} w_{1}
$$

which implies

$$
\partial_{1} \partial_{2} u=-T \partial_{2}^{2} u-\partial_{2} T \partial_{2} w_{1}+\partial_{2} w_{2}
$$

Plugging (2.5) into (2.4) yields

$$
\partial_{1}^{2} u=-\Lambda_{11}^{-1} \Lambda_{12}\left(-T \partial_{2}^{2} u-\partial_{2} T \partial_{2} w_{1}+\partial_{2} w_{2}\right)-\Lambda_{11}^{-1} \Lambda_{22} \partial_{2}^{2} u-\Lambda_{11}^{-1} R(u) .
$$

Now substituting (2.5) and (2.6) into the second equation of (2.3) we have that

$$
\begin{aligned}
\partial_{1} w_{2}= & \left\{-\Lambda_{11}^{-1} \Lambda_{12}\left(-T \partial_{2}^{2} u-\partial_{2} T \partial_{2} w_{1}+\partial_{2} w_{2}\right)-\Lambda_{11}^{-1} \Lambda_{22} \partial_{2}^{2} u-\Lambda_{11}^{-1} R(u)\right\} \\
& +T\left(-T \partial_{2}^{2} u-\partial_{2} T \partial_{2} w_{1}+\partial_{2} w_{2}\right)+\partial_{1} T \partial_{2} w_{1} \\
= & -\left(T^{2}-\Lambda_{11}^{-1} \Lambda_{12} T+\Lambda_{11}^{-1} \Lambda_{22}\right) \partial_{2}^{2} u+\left(\Lambda_{11}^{-1} \Lambda_{12} \partial_{2} T-T \partial_{2} T+\partial_{1} T\right) \partial_{2} w_{1} \\
& +\left(T-\Lambda_{11}^{-1} \Lambda_{12}\right) \partial_{2} w_{2}-\Lambda_{11}^{-1} R(u) .
\end{aligned}
$$

By the relations $\partial_{1} u=\partial_{1} w_{1}$ and $\partial_{2} u=-T^{-1} \partial_{1} w_{1}+T^{-1} w_{2}$, we can see that $R$ is a first order linear operator containing only $x_{1}$ derivative acting on $w_{1}$. More precisely, we can derive

$$
\begin{aligned}
R(u) & =\partial_{1} \Lambda_{11} \partial_{1} u+\partial_{1}{ }^{t} \Phi \partial_{2} u+\partial_{2} \Phi \partial_{1} u+\partial_{2} \Lambda_{22} \partial_{2} u \\
& =\left(\partial_{1} \Lambda_{11}+\partial_{2} \Phi\right) \partial_{1} w_{1}+\left(\partial_{2} \Lambda_{22}+\partial_{1}{ }^{t} \Phi\right)\left(-T^{-1} \partial_{1} w_{1}+T^{-1} w_{2}\right) \\
& =\left(\partial_{1} \Lambda_{11}+\partial_{2} \Phi-T^{-1} \partial_{2} \Lambda_{22}-T^{-1} \partial_{1}{ }^{t} \Phi\right) \partial_{1} w_{1}+\left(\partial_{2} \Lambda_{22}+\partial_{1}{ }^{t} \Phi\right) T^{-1} w_{2} .
\end{aligned}
$$

Replacing $R(u)$ in (2.7) by (2.8) and choosing $T$ such that

$$
T^{2}-\Lambda_{11}^{-1} \Lambda_{12} T+\Lambda_{11}^{-1} \Lambda_{22}=0 \quad \forall x \in \omega_{x_{0}}
$$

or equivalently

$$
\Lambda_{11}(-T)^{2}+\Lambda_{12}(-T)+\Lambda_{22}=0 \quad \forall x \in \omega_{x_{0}},
$$

we get from (2.3) that

$$
\left\{\begin{array}{l}
\partial_{1} w_{1}+T \partial_{2} w_{1}-w_{2}=0 \\
\Lambda_{11}^{-1}\left(\partial_{1} \Lambda_{11}+\partial_{2} \Phi-T^{-1} \partial_{2} \Lambda_{22}-T^{-1} \partial_{1}{ }^{t} \Phi\right) \partial_{1} w_{1} \\
+\partial_{1} w_{2}-\left(\Lambda_{11}^{-1} \Lambda_{12} \partial_{2} T-T \partial_{2} T+\partial_{1} T\right) \partial_{2} w_{1} \\
+\left(\Lambda_{11}^{-1} \Lambda_{12}-T\right) \partial_{2} w_{2}+\Lambda_{11}^{-1}\left(\partial_{2} \Lambda_{22}+\partial_{1}{ }^{t} \Phi\right) T^{-1} w_{2}=0
\end{array}\right.
$$

Equivalently, in the matrix form, we have that

$$
A \partial_{1} W+B \partial_{2} W+F W=0
$$

where

$$
\begin{gathered}
A=\left[\begin{array}{cc}
I & 0 \\
\Lambda_{11}^{-1}\left(\partial_{1} \Lambda_{11}+\partial_{2} \Phi-T^{-1} \partial_{2} \Lambda_{22}-T^{-1} \partial_{1}{ }^{t} \Phi\right) & I
\end{array}\right], \\
B=\left[\begin{array}{cc}
T & 0 \\
-\left(\Lambda_{11}^{-1} \Lambda_{12} \partial_{2} T-T \partial_{2} T+\partial_{1} T\right) & \Lambda_{11}^{-1} \Lambda_{12}-T
\end{array}\right],
\end{gathered}
$$

and

$$
F=\left[\begin{array}{cc}
0 & -I \\
0 & \Lambda_{11}^{-1}\left(\partial_{2} \Lambda_{22}+\partial_{1}{ }^{t} \Phi\right) T^{-1}
\end{array}\right] .
$$

The matrix $A$ is obviously invertible. We at once deduce the following first elliptic system from (2.10):

$$
\partial_{1} W+N \partial_{2} W+M W=0
$$


with

where

$$
N=A^{-1} B=\left[\begin{array}{cc}
T & 0 \\
n & \Lambda_{11}^{-1} \Lambda_{12}-T
\end{array}\right] \quad \text { and } \quad M=A^{-1} F
$$

$n=-\Lambda_{11}^{-1}\left(\partial_{1} \Lambda_{11}+\partial_{2} \Phi-T^{-1} \partial_{2} \Lambda_{22}-T^{-1} \partial_{1}{ }^{t} \Phi\right) T-\left(\Lambda_{11}^{-1} \Lambda_{12} \partial_{2} T-T \partial_{2} T+\partial_{1} T\right)$.

It should be noted that $n(x)$ is in $L^{\infty}\left(\omega_{x_{0}}\right)$ and so is $M$.

Now we are at a position to discuss the solvability of (2.9) and the Lipschitz diagonalizabilities of $T$ and $\Lambda_{11}^{-1} \Lambda_{12}-T$.

Proposition 2.1. Let $\omega_{x_{0}} \subset \Omega$ be a sufficiently small open neighborhood of $x_{0}$ and let $(\theta(x), z(x))$ be a local eigenpair of the quadratic pencil

$$
\Lambda_{11} p^{2}+\Lambda_{12} p+\Lambda_{22}
$$

i.e.

$$
\left(\Lambda_{11} \theta^{2}+\Lambda_{12} \theta+\Lambda_{22}\right) z=0 \quad \forall x \in \omega_{x_{0}} .
$$

Assume that $\theta(x), z(x)$ are Lipschitz and the matrix function $[z, \bar{z}]$ is nonsingular for all $x \in \omega_{x_{0}}$. Then by choosing

$$
-T=[z, \bar{z}] \operatorname{diag}(\theta, \bar{\theta})[z, \bar{z}]^{-1}
$$

we have that $T(x)$ satisfies (2.9) and is Lipschitz diagonalizable. Moreover, by possibly shrinking the neighborhood $\omega_{x_{0}}$, there exist a Lipschitz invertible matrix $Q(x)$ and a Lipschitz diagonal matrix $\operatorname{diag}(\rho, \bar{\rho})$ such that

$$
Q(x)^{-1}\left(\Lambda_{11}^{-1} \Lambda_{12}-T\right) Q(x)=\operatorname{diag}(\rho(x), \bar{\rho}(x)) \quad \forall x \in \omega_{x_{0}} .
$$

Proof. By the strong ellipticity condition (2.1) and the fact that $\Lambda_{11}, \Lambda_{12}, \Lambda_{22}$ are real, we can see that the eigenvalues of the quadratic pencil $\Lambda_{11} p^{2}+\Lambda_{12} p+\Lambda_{22}$ are all genuine complex values (with nonvanishing imaginary parts) and appear in conjugate pairs. Therefore, if $(\theta, z)$ is an eigenpair of the quadratic pencil, then so is $(\bar{\theta}, \bar{z})$, i.e.

$$
\left(\Lambda_{11} \bar{\theta}^{2}+\Lambda_{12} \bar{\theta}+\Lambda_{22}\right) \bar{z}=0
$$

Clearly $\bar{\theta}(x)$ and $\bar{z}(x)$ are all Lipschitz. Let $T$ be defined by (2.13); then we have that

$\left(\Lambda_{11}(-T)^{2}+\Lambda_{12}(-T)+\Lambda_{22}\right)[z, \bar{z}]=\left[\left(\Lambda_{11} \theta^{2}+\Lambda_{12} \theta+\Lambda_{22}\right) z,\left(\Lambda_{11} \bar{\theta}^{2}+\Lambda_{12} \bar{\theta}+\Lambda_{22}\right) \bar{z}\right]=0$, which implies (2.9).

Having found $T$ satisfying (2.9), we observe that

$$
\begin{aligned}
& \operatorname{det}\left(T-\Lambda_{11}^{-1} \Lambda_{12}-p I\right) \operatorname{det}(-T-p I) \\
& =\operatorname{det}\left(\left(T-\Lambda_{11}^{-1} \Lambda_{12}-p I\right)(-T-p I)\right) \\
& =\operatorname{det}\left(p^{2} I+\Lambda_{11}^{-1} \Lambda_{12} p+\Lambda_{11}^{-1} \Lambda_{12} T-T^{2}\right) \\
& =\operatorname{det}\left(p^{2} I+\Lambda_{11}^{-1} \Lambda_{12} p+\Lambda_{11}^{-1} \Lambda_{22}\right) \\
& =\operatorname{det}\left(\Lambda_{11}^{-1}\right) \operatorname{det}\left(\Lambda_{11} p^{2}+\Lambda_{12} p+\Lambda_{22}\right) .
\end{aligned}
$$

It is easily seen from (2.14) that if $\{\theta, \bar{\theta},-\rho,-\bar{\rho}\}$ are eigenvalues of the quadratic pencil $\Lambda_{11} p^{2}+\Lambda_{12} p+\Lambda_{22}$, then $\{\rho, \bar{\rho}\}$ are eigenvalues of $\Lambda_{11}^{-1} \Lambda_{12}-T$. Note that $\rho(x)$ is a genuine complex function and, hence, $\rho(x)$ and $\bar{\rho}(x)$ are distinct. In other words, $\rho(x)$ and $\bar{\rho}(x)$ are simple eigenvalues of the matrix $\Lambda_{11}^{-1} \Lambda_{12}-T$. Note that the matrix $\Lambda_{11}^{-1} \Lambda_{12}-T$ is not continuously differentiable. So we cannot use the classical implicit function theorem to conclude that $\rho(x)$ is Lipschitz. However, 
since $\rho(x)$ and $\bar{\rho}(x)$ are simple roots of a polynomial with Lipschitz coefficients, we can apply a generalized version of the implicit function theorem proved by Hildebrandt and Graves 7 ( or see [22, Theorem 4.B]) to get that $\rho(x)$ and $\bar{\rho}(x)$ are indeed Lipschitz in a possibly smaller $\omega_{x_{0}}$. Now let $\tilde{q}_{1}$ be a constant vector so that $\left[\left(\Lambda_{11}^{-1} \Lambda_{12}-T\right)\left(x_{0}\right)-\bar{\rho}\left(x_{0}\right) I\right] \tilde{q}_{1} \neq 0$; then $q_{1}(x)=\left[\left(\Lambda_{11}^{-1} \Lambda_{12}-T\right)(x)-\bar{\rho}(x) I\right] \tilde{q}_{1} \neq 0$ and $\left[\left(\Lambda_{11}^{-1} \Lambda_{12}-T\right)(x)-\rho(x) I\right] q_{1}(x)=0$ for all $x \in \omega_{x_{0}}$ (possibly smaller). Note that $q_{1}(x)$ is as smooth as $\Lambda_{11}^{-1} \Lambda_{12}-T$ and $\bar{\rho}$. Repeating the same argument for $\bar{\rho}$, we can construct $q_{2}(x)$, which is an eigenvector associated with $\bar{\rho}(x)$. Since $\rho(x)$ and $\bar{\rho}(x)$ are distinct in $\omega_{x_{0}}, Q(x)=\left[q_{1}, q_{2}\right](x)$ must be nonsingular there (see similar arguments in [8]).

Now we provide an example showing that the assumptions given in Proposition 2.1 are in fact generic.

Example 1. Consider the two-dimensional orthotropic medium where the associated matrices $\Lambda_{11}, \Lambda_{12}$, and $\Lambda_{22}$ are defined as

$$
\Lambda_{11}=\left[\begin{array}{cc}
C_{11} & 0 \\
0 & C_{66}
\end{array}\right], \Lambda_{12}=\left[\begin{array}{cc}
0 & C_{12}+C_{66} \\
C_{12}+C_{66} & 0
\end{array}\right]
$$

and

$$
\Lambda_{22}=\left[\begin{array}{cc}
C_{66} & 0 \\
0 & C_{22}
\end{array}\right] \text {. }
$$

Note that $C_{11}, C_{22}, C_{66}$ are all positive. The related quadratic pencil is now written as

$$
\left[\begin{array}{ll}
C_{11} p^{2}+C_{66} & \left(C_{12}+C_{66}\right) p \\
\left(C_{12}+C_{66}\right) p & C_{66} p^{2}+C_{22}
\end{array}\right] .
$$

We can show that if all $C$ 's are locally Lipschitz functions and satisfy the relation $(2.15)$

$$
\left(C_{11} C_{22}-C_{12}^{2}-2 C_{12} C_{66}\right)^{2}-4 C_{11} C_{22} C_{66}^{2}=: \Delta>0 \text { and } C_{12}+C_{66} \neq 0 \quad \forall x \in \Omega,
$$

or

$$
\Delta<0, C_{12}+C_{66} \neq 0, \text { and } \sqrt{C_{11} C_{22}}-C_{66} \neq 0 \quad \forall x \in \Omega,
$$

then the assumptions in Proposition 2.1 hold true. To verify this, we first observe that if $\Delta \neq 0$, then

$$
\begin{aligned}
\operatorname{det} & {\left[\begin{array}{ll}
C_{11} p^{2}+C_{66} & \left(C_{12}+C_{66}\right) p \\
\left(C_{12}+C_{66}\right) p & C_{66} p^{2}+C_{22}
\end{array}\right] } \\
& =C_{11} C_{66} p^{4}+\left[\left(C_{11} C_{22}+C_{66}^{2}\right)-\left(C_{12}+C_{66}\right)^{2}\right] p^{2}+C_{22} C_{66}=0
\end{aligned}
$$

gives rise to four distinct roots, which are locally Lipschitz. If $\Delta>0$, then all roots are purely imaginary ( $p^{2}$ is real-valued). Now let $\theta(x)$ be any root (eigenvalue) and choose the associated eigenvector

$$
z(x)=\left[\begin{array}{c}
1 \\
-\frac{\left(C_{11} \theta^{2}+C_{66}\right)}{\left(C_{12}+C_{66}\right) \theta}
\end{array}\right] .
$$

Then it is easy to see that $[z, \bar{z}]$ is nonsingular because the second component of $z$ has a nonvanishing imaginary part. Thus, we have verified condition (2.15).

Now we look at condition (2.16). Let $\theta(x)$ and $z(x)$ be chosen as above. In this case, $\theta^{2}$ is a complex-valued function (with nonvanishing real and imaginary parts) and so is $\theta$. To see that $[z, \bar{z}]$ is nonsingular, it suffices to show that $\frac{\left(C_{11} \theta^{2}+C_{66}\right)}{\left(C_{12}+C_{66}\right)}$ has 
a nonvanishing imaginary part. We prove this by contradictory arguments. Assume that $\frac{\left(C_{11} \theta^{2}+C_{66}\right)}{\left(C_{12}+C_{66}\right) \theta}$ becomes real-valued at some point $x_{0}$. Then we must have that

$$
\frac{\left(C_{11} \theta^{2}+C_{66}\right)}{\left(C_{12}+C_{66}\right) \theta}=\frac{\left(C_{11} \bar{\theta}^{2}+C_{66}\right)}{\left(C_{12}+C_{66}\right) \bar{\theta}} \quad \text { at } x_{0}
$$

which leads to

$$
C_{11}|\theta|^{2}=C_{66} \quad \text { at } x_{0}
$$

Note that $|\theta|^{2}=\sqrt{\frac{C_{22}}{C_{11}}}$. Therefore, we have that $\sqrt{C_{11} C_{22}}=C_{66}$ at $x_{0}$, which is a contradiction.

In next example we consider the isotropic case. It turns out that the assumptions in Proposition 2.1 hold without further restriction on the Lamé coefficients other than the usual strong ellipticity condition.

Example 2. Let the medium be isotropic; then we have that

$$
C_{11}=C_{22}=\lambda+2 \mu, C_{12}=\lambda, C_{66}=\mu
$$

in Example 1, where $\lambda(x)$ and $\mu(x)$ are locally Lipschitz functions in $\Omega$. Assume that $\mu(x)>0$ and $\lambda(x)+2 \mu(x)>0$ for all $x \in \Omega$. Then the eigenvalues of the related quadratic pencil are $\pm i$ (repeated) with associated eigenvectors ${ }^{t}[1, \mp i]$. Therefore, if we choose $\theta=i$ and $z={ }^{t}[1,-i]$, then the assumption of Proposition [2.1] is clearly satisfied. Furthermore, we can compute that

$$
\Lambda_{11}^{-1} \Lambda_{12}-T=\left[\begin{array}{cc}
0 & \frac{-\mu}{\lambda+2 \mu} \\
\frac{\lambda+2 \mu}{\mu} & 0
\end{array}\right] \text {. }
$$

So when we take

$$
Q=\left[\begin{array}{cc}
1 & 1 \\
-\frac{\lambda+2 \mu}{\mu} i & \frac{\lambda+2 \mu}{\mu} i
\end{array}\right]
$$

we get that

$$
Q^{-1}\left(\Lambda_{11}^{-1} \Lambda_{22}-T\right) Q=\operatorname{diag}(i,-i) .
$$

Now, in view of Proposition 2.1, we define a Lipschitz invertible matrix

$$
P(x)=\left[\begin{array}{cc}
{[z, \bar{z}]} & 0 \\
0 & Q
\end{array}\right]
$$

and set $W=P V$ in $\omega_{x_{0}}$. Then from (2.11) we obtain that

$$
\partial_{1} V+\widetilde{N} \partial_{2} V+\widetilde{M} V=0 \quad\left(\text { a.e. in } \omega_{x_{0}}\right),
$$

where

and

$$
\widetilde{N}=\left[\begin{array}{cc}
\operatorname{diag}(-\mu,-\bar{\mu}) & 0 \\
n & \operatorname{diag}(\rho, \bar{\rho})
\end{array}\right]
$$

$$
\widetilde{M}=P^{-1} \partial_{1} P+P^{-1} N \partial_{2} P+P^{-1} M P \in L^{\infty}\left(\omega_{x_{0}}\right) .
$$

As we have mentioned in the Introduction, $N$ is not necessarily Lipschitz diagonalizable since $-\mu(x)$ and $\rho(x)$ may coincide at some points in $\omega_{x_{0}}$, and $n(x)$ is merely essentially bounded. Now, to prove the UCP for (1.2), it suffices to prove that for (2.17). To do this, we will derive some Carleman estimates, which will be carried out in the following section. 
2.2. Carleman estimates. Let $L_{\lambda} v=\partial_{1} v+\lambda(x) \partial_{2} v$ be a first order scalar elliptic operator, where $\lambda(x)=\alpha(x)+i \beta(x)$ is a locally Lipschitz function and $\beta(x)$ never vanishes in $\mathbb{R}^{2}$. Given $0<r_{0}<1$, define $U=\left\{v \in C_{0}^{\infty}\left(\mathbb{R}^{2} \backslash\{0\}\right): \operatorname{supp}(v) \subset B_{r_{0}}\right\}$, where $B_{r_{0}}$ is the disk centered at the origin with radius $r_{0}$. From now on, we use $c$ or $\tilde{c}$ to denote general positive constants whose values may vary from line to line.

Theorem 2.2. There exist a constant $c>0$ and a sufficiently large number $s_{0}>0$ such that for all $v \in U$ and $s \geq s_{0}$ we have that

$$
s^{2} \int r^{-\sigma-s-2} \phi_{s}^{2}|v|^{2} d x \leq c \int r^{-\sigma} \phi_{s}^{2}\left|L_{\lambda} v\right|^{2} d x,
$$

where $r=|x|, \phi_{s}=\exp \left(r^{-s}\right)$ and $\sigma=\sigma_{s}=\sigma_{0}+\sigma_{1} s$ with $\sigma_{0}, \sigma_{1} \in \mathbb{R}$.

Proof. The proof of the theorem is motivated by Hile and Protter's paper [8] in which they considered the system of equations without the parameter $\sigma$. As in [8], we will work on a slightly different operator instead of $L_{\lambda}$. For simplicity, we denote $L:=L_{\lambda}$. Define the scalar function

$$
\eta(x)=\frac{1}{2}\left(\frac{\partial_{1} \beta}{\beta}+i \partial_{2} \beta+\alpha \frac{\partial_{2} \beta}{\beta}\right)
$$

and consider the operator

$$
\widetilde{L} v=L v+\eta v=\partial_{1} v+\lambda \partial_{2} v+\eta v .
$$

It is easily seen that there exists a constant $c>0$ such that

$$
|\widetilde{L} v| \leq|L v|+c|v| .
$$

Now we set $\psi(r)=r^{-s}-\frac{\sigma}{2} \log r$ and denote $w=e^{\psi} v$. Then we can find that

$$
\int e^{2 \psi}|\widetilde{L} v|^{2} d x=\int\left|\partial_{1} w+\lambda \partial_{2} w-\partial_{1} \psi w-\lambda \partial_{2} \psi w+\eta w\right|^{2} d x .
$$

Following the notations in 8 ] we set

$$
\begin{aligned}
& a_{1}=\partial_{1} w, a_{2}=\alpha \partial_{2} w, a_{3}=i \beta \partial_{2} w, a_{4}=-\left(\partial_{1} \psi w+\alpha \partial_{2} \psi w\right), \\
& a_{5}=-i \beta \partial_{2} \psi w, a_{6}=\frac{1}{2} \frac{\partial_{1} \beta}{\beta} w, a_{7}=\frac{1}{2} i \partial_{2} \beta w, a_{8}=\frac{1}{2}\left(\alpha \frac{\partial_{2} \beta}{\beta} w\right) .
\end{aligned}
$$

Now from (2.20) we can write

$$
\begin{aligned}
& \int e^{2 \psi}|\tilde{L} v|^{2} d x=\int\left|\sum_{j=1}^{8} a_{j}\right|^{2} d x \\
& =\int\left\{\left|a_{1}+a_{2}+a_{5}+a_{6}+a_{8}\right|^{2}+2 \operatorname{Re}\left(\left(a_{1}+a_{2}+a_{5}+a_{6}+a_{8}\right) \cdot \overline{\left(a_{3}+a_{4}+a_{7}\right)}\right)\right. \\
& \left.\quad \quad+\left|a_{3}+a_{4}+a_{7}\right|^{2}\right\} d x \\
& \geq 2 \operatorname{Re} \int\left(a_{1}+a_{2}+a_{5}+a_{6}+a_{8}\right) \cdot \overline{\left(a_{3}+a_{4}+a_{7}\right)} d x .
\end{aligned}
$$

It is readily seen that $\operatorname{Re}\left(a_{2} \bar{a}_{3}\right)=\operatorname{Re}\left(a_{5} \bar{a}_{4}\right)=\operatorname{Re}\left(a_{6} \bar{a}_{7}\right)=\operatorname{Re}\left(a_{8} \bar{a}_{7}\right)=0$ and

$$
2 \operatorname{Re}\left(a_{2} \bar{a}_{7}+a_{8} \bar{a}_{3}\right)=\operatorname{Re}\left(-i \alpha \partial_{2} \beta\left(\bar{w} \partial_{2} w+w \partial_{2} \bar{w}\right)\right)=0 .
$$

We at once deduce that

$$
\begin{aligned}
2 \operatorname{Re} \int a_{2} \bar{a}_{3} d x & =2 \operatorname{Re} \int a_{5} \bar{a}_{4} d x=2 \operatorname{Re} \int a_{6} \bar{a}_{7} d x=2 \operatorname{Re} \int a_{8} \bar{a}_{7} d x \\
& =2 \operatorname{Re} \int\left(a_{2} \bar{a}_{7}+a_{8} \bar{a}_{3}\right) d x=0 .
\end{aligned}
$$


Next, note that

$$
\begin{aligned}
\operatorname{Re}\left(\partial_{2}\left(i \beta w \partial_{1} \bar{w}\right)+\partial_{1}\left(-i \beta w \partial_{2} \bar{w}\right)\right) & =\operatorname{Re}\left(2 i \beta \partial_{2} w \partial_{1} \bar{w}+i \partial_{2} \beta w \partial_{1} \bar{w}-i \partial_{1} \beta w \partial_{2} \bar{w}\right) \\
& =2 \operatorname{Re}\left(a_{1} \bar{a}_{3}+a_{1} \bar{a}_{7}+a_{6} \bar{a}_{3}\right) .
\end{aligned}
$$

Therefore, performing the integration by parts leads to

$$
2 \operatorname{Re} \int\left(a_{1} \bar{a}_{3}+a_{1} \bar{a}_{7}+a_{6} \bar{a}_{3}\right) d x=0 .
$$

Thus, there are five terms left on the right side of (2.21) needed to be estimated, namely,

$$
2 \operatorname{Re} \int\left(a_{1} \bar{a}_{4}+a_{2} \bar{a}_{4}+a_{5}\left(\bar{a}_{3}+\bar{a}_{7}\right)+a_{6} \bar{a}_{4}+a_{8} \bar{a}_{4}\right) d x .
$$

We begin with the first term of (2.22). Using the integration by parts, we get that

$$
\begin{aligned}
2 \operatorname{Re} \int a_{1} \bar{a}_{4} d x & =-2 \operatorname{Re} \int \partial_{1} w\left(\partial_{1} \psi \bar{w}+\alpha \partial_{2} \psi \bar{w}\right) d x \\
& =-\int\left(\partial_{1} \psi \partial_{1}|w|^{2}+\alpha \partial_{2} \psi \partial_{1}|w|^{2}\right) d x \\
& =\int\left\{\left(\partial_{1}^{2} \psi+\alpha \partial_{1} \partial_{2} \psi\right)|w|^{2}+\partial_{1} \alpha \partial_{2} \psi|w|^{2}\right\} d x \\
& \geq \int\left(\partial_{1}^{2} \psi+\alpha \partial_{1} \partial_{2} \psi\right)|w|^{2} d x-c \int\left|\partial_{2} \psi\right||w|^{2} d x .
\end{aligned}
$$

Similarly, we can find that

$$
\begin{aligned}
2 \operatorname{Re} \int a_{2} \bar{a}_{4} d x & =-2 \operatorname{Re} \int \alpha \partial_{2} w\left(\partial_{1} \psi \bar{w}+\alpha \partial_{2} \psi \bar{w}\right) d x \\
& =-\int\left(\alpha \partial_{1} \psi \partial_{2}|w|^{2}+\alpha^{2} \partial_{2} \psi \partial_{2}|w|^{2}\right) d x \\
& =\int\left\{\left(\alpha \partial_{1} \partial_{2} \psi+\alpha^{2} \partial_{2}^{2} \psi\right)|w|^{2}+\left(\partial_{2} \alpha \partial_{1} \psi+\partial_{2} \alpha^{2} \partial_{2} \psi\right)|w|^{2}\right\} d x \\
& \geq \int\left(\alpha \partial_{1} \partial_{2} \psi+\alpha^{2} \partial_{2}^{2} \psi\right)|w|^{2} d x-c \int\left(\left|\partial_{2} \psi\right|+\left|\partial_{1} \psi\right|\right)|w|^{2} d x
\end{aligned}
$$

and

$$
\begin{aligned}
2 \operatorname{Re} \int a_{5} \bar{a}_{3} d x & =-2 \operatorname{Re} \int \beta^{2} \partial_{2} \psi w \partial_{2} \bar{w} d x \\
& =-\int \beta^{2} \partial_{2} \psi \partial_{2}|w|^{2} d x \\
& =\int \beta^{2} \partial_{2}^{2} \psi|w|^{2} d x+\int \partial_{2} \beta^{2} \partial_{2} \psi|w|^{2} d x \\
& \geq \int \beta^{2} \partial_{2}^{2} \psi|w|^{2} d x-c \int\left|\partial_{2} \psi\right||w|^{2} d x .
\end{aligned}
$$

Furthermore, we can estimate

$$
\left\{\begin{array}{l}
2 \operatorname{Re} \int a_{5} \bar{a}_{7} d x \geq-c \int\left|\partial_{2} \psi\right||w|^{2} d x \\
2 \operatorname{Re} \int a_{6} \bar{a}_{4} d x \geq-c \int\left(\left|\partial_{1} \psi\right|+\left|\partial_{2} \psi\right|\right)|w|^{2} d x, \\
2 \operatorname{Re} \int a_{8} \bar{a}_{4} \geq-c \int\left(\left|\partial_{1} \psi\right|+\left|\partial_{2} \psi\right|\right)|w|^{2} d x
\end{array}\right.
$$

Combining (2.23), (2.24), (2.25) and (2.26) yields

$$
\begin{aligned}
& 2 \operatorname{Re} \int\left(a_{1} \bar{a}_{4}+a_{2} \bar{a}_{4}+a_{5}\left(\bar{a}_{3}+\bar{a}_{7}\right)+a_{6} \bar{a}_{4}+a_{8} \bar{a}_{4}\right) d x \\
& \geq \int\left(\partial_{1}^{2} \psi+2 \alpha \partial_{1} \partial_{2} \psi+\left(\alpha^{2}+\beta^{2}\right) \partial_{2}^{2} \psi\right)|w|^{2} d x-c \int\left(\left|\partial_{1} \psi\right|+\left|\partial_{2} \psi\right|\right)|w|^{2} d x .
\end{aligned}
$$

Through direct computations, we obtain that

$$
\begin{aligned}
& \partial_{j} \psi=-s r^{-s-2} x_{j}-(\sigma / 2) r^{-2} x_{j}, \\
& \partial_{j}^{2} \psi=s(s+2) r^{-s-4} x_{j}^{2}-s r^{-s-2}+\sigma r^{-4} x_{j}^{2}-(\sigma / 2) r^{-2}, j=1,2,
\end{aligned}
$$

and

$$
\partial_{1} \partial_{2} \psi=s(s+2) r^{-s-4} x_{1} x_{2}+\sigma r^{-4} x_{1} x_{2} .
$$

Therefore, by taking $s$ sufficiently large, we can get from (2.27) that

$$
\begin{aligned}
& 2 \operatorname{Re} \int\left(a_{1} \bar{a}_{4}+a_{2} \bar{a}_{4}+a_{5}\left(\bar{a}_{3}+\bar{a}_{7}\right)+a_{6} \bar{a}_{4}+a_{8} \bar{a}_{4}\right) d x \\
& \geq s^{2} \int r^{-s-4}\left(x_{1}^{2}+2 \alpha x_{1} x_{2}+\left(\alpha^{2}+\beta^{2}\right) x_{2}^{2}\right)|w|^{2} d x-c s \int r^{-s-2}|w|^{2} d x .
\end{aligned}
$$


Note that

$$
x_{1}^{2}+2 \alpha x_{1} x_{2}+\left(\alpha^{2}+\beta^{2}\right) x_{2}^{2}=\left|x_{1}+\lambda x_{2}\right|^{2} .
$$

By easy computations, we have the following identity:

$$
\left(1+|\lambda|^{2}\right)\left|x_{1}+\lambda x_{2}\right|^{2}=\beta^{2}\left(x_{1}^{2}+x_{2}^{2}\right)+\left(x_{1}+\alpha x_{2}\right)^{2}+\left(\alpha x_{1}+\alpha^{2} x_{2}+\beta^{2} x_{2}^{2}\right)^{2},
$$

which leads to

$$
\left|x_{1}+\lambda x_{2}\right|^{2} \geq \frac{\beta^{2} r^{2}}{1+\sup _{B_{r_{0}}}|\lambda|^{2}} \quad \forall x \in B_{r_{0}} .
$$

In view of the assumption on the imaginary part of $\lambda$, we immediately get that

$$
|\beta(x)| \geq \delta>0 \quad \forall x \in B_{r_{0}} .
$$

Substituting (2.29), (2.30), (2.31) into (2.28) and taking $s$ large enough, we conclude that

$$
2 \operatorname{Re} \int\left(a_{1} \bar{a}_{4}+a_{2} \bar{a}_{4}+a_{5}\left(\bar{a}_{3}+\bar{a}_{7}\right)+a_{6} \bar{a}_{4}+a_{8} \bar{a}_{4}\right) d x \geq c s^{2} \int r^{-s-2}|w|^{2} d x
$$

and, hence,

$$
\begin{aligned}
\int e^{2 \psi}|\widetilde{L} v|^{2} d x & =\int r^{-\sigma} \phi_{s}^{2}|\widetilde{L} v|^{2} d x \geq c s^{2} \int r^{-s-2} r^{2 \psi}|v|^{2} d x \\
& =c s^{2} \int r^{-\sigma-s-2} \phi_{s}^{2}|v|^{2} d x
\end{aligned}
$$

Finally, in view of (2.19), we obtain the estimate (2.18).

To handle the possible nonzero off-diagonal block in $\tilde{N}$, we need another Carleman estimate.

Theorem 2.3. There exist a constant $c>0$ and a sufficiently large number $s_{0}>0$ such that for all $v \in U$ and $s \geq s_{0}$ we have the following estimate:

$$
\int r^{-\sigma} \phi_{s}^{2}\left|\partial_{2} v\right|^{2} d x \leq c \int r^{-\sigma} \phi_{s}^{2}\left(\left|L_{\lambda} v\right|^{2}+s^{2} r^{-2 s-2}|v|^{2}\right) d x .
$$

Proof. To begin with, we set $v=v_{R}+i v_{I}$ and $L_{\lambda}=L$. Now we can compute (2.33)

$$
\begin{aligned}
& \int r^{-\sigma} \phi_{s}^{2}|L v|^{2} d x \\
& =\int r^{-\sigma} \phi_{s}^{2}\left\{\left|\partial_{1} v\right|^{2}+|\lambda|^{2}\left|\partial_{2} v\right|^{2}+2 \operatorname{Re}\left(\partial_{1} v \overline{\lambda \partial_{2} v}\right)\right\} d x \\
& =\int r^{-\sigma} \phi_{s}^{2}\left(\left|\partial_{1} v\right|^{2}+\left(\alpha^{2}+\beta^{2}\right)\left|\partial_{2} v\right|^{2}\right) d x+2 \int r^{-\sigma} \phi_{s}^{2} \alpha\left(\partial_{1} v_{R} \partial_{2} v_{R}+\partial_{1} v_{I} \partial_{2} v_{I}\right) d x \\
& \quad-2 \int r^{-\sigma} \phi_{s}^{2} \beta\left(\partial_{1} v_{R} \partial_{2} v_{I}-\partial_{1} v_{I} \partial_{2} v_{R}\right) d x .
\end{aligned}
$$

Using the inequality

$$
|a b| \leq\left(\varepsilon a^{2}+\varepsilon^{-1} b^{2}\right) / 2 \text { for any } \varepsilon>0,
$$

we have that

$$
\begin{aligned}
& \left|\int r^{-\sigma} \phi_{s}^{2} \alpha\left(\partial_{1} v_{R} \partial_{2} v_{R}+\partial_{1} v_{I} \partial_{2} v_{I}\right) d x\right| \\
& \leq \frac{1}{2} \int r^{-\sigma} \phi_{s}^{2}\left\{\left(\partial_{1} v_{R}\right)^{2}+\left(\partial_{1} v_{I}\right)^{2}\right\} d x+\frac{1}{2} \int r^{-\sigma} \phi_{s}^{2} \alpha^{2}\left\{\left(\partial_{2} v_{R}\right)^{2}+\left(\partial_{2} v_{I}\right)^{2}\right\} d x \\
& =\frac{\varepsilon_{1}}{2} \int r^{-\sigma} \phi_{s}^{2}\left|\partial_{1} v\right|^{2} d x+\frac{1}{2} \varepsilon_{1}^{-1} \int r^{-\sigma} \phi_{s}^{2} \alpha^{2}\left|\partial_{2} v\right|^{2} d x
\end{aligned}
$$


where $\varepsilon_{1}>0$ will be chosen later. On the other hand, from the integration by parts, one can easily derive that

$$
\begin{aligned}
& \left|\int r^{-\sigma} \phi_{s}^{2} \beta\left(\partial_{1} v_{R} \partial_{2} v_{I}-\partial_{1} v_{I} \partial_{2} v_{R}\right) d x\right| \\
& =\left|-\int \partial_{1}\left(r^{-\sigma} \phi_{s}^{2} \beta\right) \partial_{2} v_{I} v_{R} d x+\int \partial_{2}\left(r^{-\sigma} \phi_{s}^{2} \beta\right) \partial_{1} v_{I} v_{R} d x\right| \\
& \leq\left|\int\left\{\left(-\sigma r^{-\sigma-2}-2 s r^{-\sigma-s-2}\right) \phi_{s}^{2} x_{1} \beta \partial_{2} v_{I} v_{R}+r^{-\sigma} \phi_{s}^{2} \partial_{1} \beta \partial_{2} v_{I} v_{R}\right\} d x\right| \\
& \quad+\left|\int\left\{\left(-\sigma r^{-\sigma-2}-2 s r^{-\sigma-s-2}\right) \phi_{s}^{2} x_{2} \beta \partial_{1} v_{I} v_{R}+r^{-\sigma} \phi_{s}^{2} \partial_{2} \beta \partial_{1} v_{I} v_{R}\right\} d x\right| \\
& \leq\left|\int(-\sigma) r^{-\sigma-2} \phi_{s}^{2} x_{1} \beta \partial_{2} v_{I} v_{R} d x\right|+\left|\int(-2 s) r^{-\sigma-s-2} \phi_{s}^{2} x_{1} \beta \partial_{2} v_{I} v_{R} d x\right| \\
& \quad+\left|\int(-\sigma) r^{-\sigma-2} \phi_{s}^{2} x_{2} \beta \partial_{1} v_{I} v_{R} d x\right|+\left|\int(-2 s) r^{-\sigma-s} \phi_{s}^{2} x_{2} \beta \partial_{1} v_{I} v_{R} d x\right| \\
& \quad+\left|\int r^{-\sigma} \phi_{s}^{2} \partial_{1} \beta \partial_{2} v_{I} v_{R} d x\right|+\left|\int r^{-\sigma} \phi_{s}^{2} \partial_{2} \beta \partial_{1} v_{I} v_{R} d x\right| \\
& \leq \frac{1}{2} \varepsilon_{2} \int r^{-\sigma} \phi_{s}^{2} \beta^{2}\left(\partial_{2} v_{I}\right)^{2} d x+\frac{1}{2}|\sigma|^{2} \varepsilon_{2}^{-1} \int r^{-\sigma-4} \phi_{s}^{2} x_{1}^{2} v_{R}^{2} d x \\
& \quad+\frac{1}{2} \varepsilon_{2} \int r^{-\sigma} \phi_{s}^{2} \beta^{2}\left(\partial_{2} v_{I}\right)^{2} d x+2 s^{2} \varepsilon_{2}^{-1} \int r^{-\sigma-2 s-4} \phi_{s}^{2} x_{1}^{2} v_{R}^{2} d x \\
& \quad+\frac{1}{2} \varepsilon_{2} \int r^{-\sigma} \phi_{s}^{2}\left(\partial_{1} v_{I}\right)^{2} d x+\frac{1}{2}|\sigma|^{2} \varepsilon_{2}^{-1} \int r^{-\sigma-4} \phi_{s}^{2} x_{2}^{2} \beta^{2} v_{R}^{2} d x \\
& \quad+\frac{1}{2} \varepsilon_{2} \int r^{-\sigma} \phi_{s}^{2}\left(\partial_{1} v_{I}\right)^{2} d x+2 s^{2} \varepsilon_{2}^{-1} \int r^{-\sigma-2 s-4} \phi_{s}^{2} x_{2}^{2} \beta^{2} v_{R}^{2} d x \\
& \quad+\frac{1}{2} \varepsilon_{2} \int r^{-\sigma} \phi_{s}^{2} \beta^{2}\left(\partial_{2} v_{I}\right)^{2} d x+\frac{1}{2} \varepsilon_{2}^{-1} \int r^{-\sigma} \phi_{s}^{2}\left(\frac{\partial_{1} \beta}{\beta}\right)^{2} v_{R}^{2} d x \\
& \quad+\frac{1}{2} \varepsilon_{2} \int r^{-\sigma} \phi_{s}^{2}\left(\partial_{1} v_{I}\right)^{2} d x+\frac{1}{2} \varepsilon_{2}^{-1} \int r^{-\sigma} \phi_{s}^{2}\left(\partial_{1} \beta\right)^{2} v_{R}^{2} d x \\
& \leq \frac{3 \varepsilon_{2}}{2} \int r^{-\sigma} \phi_{s}^{2}\left\{\left(\partial_{1} v_{I}\right)^{2}+\beta^{2}\left(\partial_{2} v_{I}\right)^{2}\right\} d x+c s^{2} \varepsilon_{2}^{-1} \int r^{-\sigma-2 s-2} \phi_{s}^{2} v_{R}^{2} d x,
\end{aligned}
$$

where $\varepsilon_{2}>0$ will be determined below. In deriving (2.36), we once again used the inequality (2.34). Combining (2.33), (2.35), (2.36) and choosing $3 \varepsilon_{2}=1-\varepsilon_{1}$ with $0<\varepsilon_{1}<1$, we obtain that

$$
\begin{aligned}
& \int r^{-\sigma} \phi_{s}^{2}|L v|^{2} d x \\
& \geq\left(1-\varepsilon_{1}-3 \varepsilon_{2}\right) \int r^{-\sigma} \phi_{s}^{2}\left|\partial_{1} v\right|^{2} d x+\int r^{-\sigma} \phi_{s}^{2}\left(\alpha^{2}+\beta^{2}-\varepsilon_{1}^{-1} \alpha^{2}-3 \varepsilon_{2} \beta^{2}\right)\left|\partial_{2} v\right|^{2} d x \\
& \quad-2 c \varepsilon_{2}^{-1} s^{2} \int r^{-\sigma-2 s-2} \phi_{s}^{2}|v|^{2} d x \\
& \geq \int r^{-\sigma} \phi_{s}^{2}\left(\left(1-\varepsilon_{1}^{-1}\right) \alpha^{2}+\varepsilon_{1} \beta^{2}\right)\left|\partial_{2} v\right|^{2} d x-6 c\left(1-\varepsilon_{1}\right)^{-1} s^{2} \int r^{-\sigma-2 s-2} \phi_{s}^{2}|v|^{2} d x
\end{aligned}
$$

Since $|\beta(x)| \geq \delta>0$ for all $x \in B_{r_{0}}$, we can find an $\varepsilon_{1}$ sufficiently close to 1 such that

$$
\left(1-\varepsilon_{1}^{-1}\right) \alpha^{2}+\varepsilon_{1} \beta^{2} \geq \widetilde{\delta}>0 \quad \forall x \in B_{r_{0}} .
$$

Now the Carleman estimate (2.32) follows from (2.37) and (2.38).

2.3. Proof of the UCP. Here we will prove a weaker version of UCP for (1.2). Namely, we would like to show that a solution $u$ of (1.2) that vanishes at one point in $\Omega$ of any exponential order must vanish in $\Omega$ provided that the assumption of Proposition 2.1 holds near every point in $\Omega$. It is clear that the UCP follows from this version of continuation property. We say that $u$ vanishes at $x_{0}$ of any exponential order if

$$
\lim _{\left|x-x_{0}\right| \rightarrow 0} \exp \left(\left|x-x_{0}\right|^{-s}\right) u(x)=0 \quad \forall s>0 .
$$

Theorem 2.4. Assume that the assumptions of Proposition 2.1 are satisfied near every point in $\Omega$. Let $u$ vanish at some $x_{0} \in \Omega$ of any exponential order; then $u \equiv 0$ in $\Omega$.

Proof. Without loss of generality, we assume $x_{0}=0$. Let the assumptions of Proposition 2.1 hold in $\omega_{0} \subset \Omega$, where $\omega_{0}$ is a small neighborhood of 0 . Then we can convert (1.2) into (2.17) in $\omega_{0}$. Define $r_{0}=\min \left\{1 / 2\right.$, dist $\left.\left(0, \partial \omega_{0}\right)\right\}$. Now let $\chi \in C_{0}^{\infty}\left(\mathbb{R}^{2}\right)$ be a cut-off function satisfying $0 \leq \chi \leq 1,\left.\chi\right|_{B_{r_{0} / 2}}=1$ and $\operatorname{supp}(\chi) \subset B_{r_{0}}$. Define $\widetilde{V}={ }^{t}\left(\tilde{v}_{1}, \tilde{v}_{2}, \tilde{v}_{3}, \tilde{v}_{4}\right)=\chi V$. Then from (2.17), we get that

$$
\partial_{1} \widetilde{V}+\widetilde{N} \partial_{2} \widetilde{V}=-\widetilde{M} \widetilde{V}+G \text {, }
$$


where $G={ }^{t}\left(g_{1}, g_{2}, g_{3}, g_{4}\right) \in L^{2}(\Omega)$ is supported in $B_{r_{0}} \backslash B_{r_{0} / 2}$. Denote $\lambda_{1}=\bar{\lambda}_{2}=$ $-\theta$ and $\lambda_{3}=\bar{\lambda}_{4}=\rho$. We set

$$
\begin{aligned}
& H=\gamma \int r^{-s} \phi_{s}^{2}\left(\left|L_{\lambda_{1}} \tilde{v}_{1}\right|^{2}+\left|L_{\lambda_{2}} \tilde{v}_{2}\right|^{2}\right) d x+\int \phi_{s}^{2}\left(\left|L_{\lambda_{3}} \tilde{v}_{3}\right|^{2}+\left|L_{\lambda_{4}} \tilde{v}_{4}\right|^{2}\right) d x, \\
& K=\gamma \int r^{-s} \phi_{s}^{2}\left(\left|\tilde{v}_{1}\right|^{2}+\left|\tilde{v}_{2}\right|^{2}\right) d x+\int \phi_{s}^{2}\left(\left|\tilde{v}_{3}\right|^{2}+\left|\tilde{v}_{4}\right|^{2}\right) d x, \\
& J=\gamma \int r^{-s} \phi_{s}^{2}\left(\left|g_{1}\right|^{2}+\left|g_{2}\right|^{2}\right) d x+\int \phi_{s}^{2}\left(\left|g_{3}\right|^{2}+\left|g_{4}\right|^{2}\right) d x,
\end{aligned}
$$

where $\gamma>0$ will be chosen later. It follows from (2.39) that

$$
H \leq c\left(K+J+\int \phi_{s}^{2}\left(\left|\partial_{2} \tilde{v}_{1}\right|^{2}+\left|\partial_{2} \tilde{v}_{2}\right|^{2}\right) d x\right) .
$$

By the standard approximation argument, we can see that $\tilde{v}_{j}$ satisfies estimates (2.18) and (2.32) for all $1 \leq j \leq 4$. Now replacing the last term of (2.40) by the estimate (2.32) for $L_{\lambda_{1}}$ and $L_{\lambda_{2}}$ with $\sigma=0$, we have that

$$
H \leq c\left(K+J+\int \phi_{s}^{2}\left(\left|L_{\lambda_{1}} \tilde{v}_{1}\right|^{2}+\left|L_{\lambda_{2}} \tilde{v}_{2}\right|^{2}\right) d x+s^{2} \int r^{-2 s-2} \phi_{s}^{2}\left(\left|\tilde{v}_{1}\right|^{2}+\left|\tilde{v}_{2}\right|^{2}\right) d x\right) .
$$

Substituting the estimate (2.18) for $L_{\lambda_{1}}$ and $L_{\lambda_{2}}$ with $\sigma=-s$ into the last term of (2.41) leads to

$$
H \leq c\left(K+J+\int r^{-s} \phi_{s}^{2}\left(\left|L_{\lambda_{1}} \tilde{v}_{1}\right|^{2}+\left|L_{\lambda_{2}} \tilde{v}_{2}\right|^{2}\right) d x\right)
$$

In deriving (2.42), we also use the obvious inequality $r^{-s}>1$ for any $0<r<1$ and $s>0$. Taking $\gamma \gg 1$, we can absorb the last term of (2.42) and get

$$
H \leq c(K+J) .
$$

From now on we fix the parameter $\gamma$.

Next repeatedly using $\sigma=s$ in (2.18) for $L_{\lambda_{1}}, L_{\lambda_{2}}$ and $\sigma=0$ in (2.18) for $L_{\lambda_{3}}$, $L_{\lambda_{4}}$, we find that

$$
\begin{aligned}
& s^{2} \int r^{-2 s-2} \phi_{s}^{2}\left(\left|\tilde{v}_{1}\right|^{2}+\left|\tilde{v}_{2}\right|^{2}\right) d x+s^{2} \int r^{-s-2} \phi_{s}^{2}\left(\left|\tilde{v}_{3}\right|^{2}+\left|\tilde{v}_{4}\right|^{2}\right) d x \\
& \leq c\left(\int r^{-s} \phi_{s}^{2}\left(\left|L_{\lambda_{1}} \tilde{v}_{1}\right|^{2}+\left|L_{\lambda_{2}} \tilde{v}_{2}\right|^{2}\right) d x+\int \phi_{s}^{2}\left(\left|L_{\lambda_{3}} \tilde{v}_{3}\right|^{2}+\left|L_{\lambda_{4}} \tilde{v}_{4}\right|^{2}\right) d x\right) \\
& \leq c H \leq c(K+J)
\end{aligned}
$$

In view of the inequalities $1<r^{-s}<r^{-s-2}<r^{-2 s-2}$ for $0<r<1$, by taking $s \gg 1$, we get from (2.44) that

$$
\begin{aligned}
& s^{2} \int r^{-s-2} \phi_{s}^{2}|\widetilde{V}|^{2} d x \\
& \leq s^{2} \int r^{-2 s-2} \phi_{s}^{2}\left(\left|\tilde{v}_{1}\right|^{2}+\left|\tilde{v}_{2}\right|^{2}\right) d x+s^{2} \int r^{-s-2} \phi_{s}^{2}\left(\left|\tilde{v}_{3}\right|^{2}+\left|\tilde{v}_{4}\right|^{2}\right) d x \\
& \leq c\left(\int r^{-s} \phi_{s}^{2}\left(\left|\tilde{v}_{1}\right|^{2}+\left|\tilde{v}_{2}\right|^{2}\right) d x+\int \phi_{s}^{2}\left(\left|\tilde{v}_{3}\right|^{2}+\left|\tilde{v}_{4}\right|^{2}\right) d x\right) \\
& \quad+c\left(\int r^{-s} \phi_{s}^{2}\left(\left|g_{1}\right|^{2}+\left|g_{2}\right|^{2}\right) d x+\int \phi_{s}^{2}\left(\left|g_{3}\right|^{2}+\left|g_{4}\right|^{2}\right) d x\right) \\
& \leq c \int r^{-s-2} \phi_{s}^{2}|\widetilde{V}|^{2} d x+c \int r^{-s-2} \phi_{s}^{2}|G|^{2} d x \\
& \leq \tilde{c} \int r^{-s-2} \phi_{s}^{2}|G|^{2} d x,
\end{aligned}
$$

which leads to

$$
s^{2} \int_{B_{r_{0} / 2}} r^{-s-2} \phi_{s}^{2}|\widetilde{V}|^{2} d x \leq c \int_{B_{r_{0}} \backslash B_{r_{0} / 2}} r^{-s-2} \phi_{s}^{2}|G|^{2} d x .
$$

Since $r^{-s-2} \phi_{s}^{2}$ is a strictly decreasing function, (2.45) implies that

$$
s^{2} \int_{B_{r_{0} / 2}} \tilde{V} d x \leq c \int_{B_{r_{0}} \backslash B_{r_{0} / 2}}|G|^{2} d x<\infty
$$


and thus $\widetilde{V}=V=0$, i.e. $u=0$, on $B_{r_{0} / 2}$ if we choose $s$ sufficiently large. Now the standard arguments imply that the set $\{x \in \Omega: u=0\}$ is open and closed in $\Omega$. Since $\Omega$ is connected, we must have $u \equiv 0$ in $\Omega$.

In view of Example 2, we know that the assumption of Proposition 2.1 holds automatically for the isotropic elastic tensor. So from Theorem 2.4 we immediately conclude that the UCP is valid for the isotropic elasticity system with locally Lipschitz Lamé coefficients.

Corollary 2.5. Let $C(x)$ be isotropic, i.e.

$$
C_{i j k l}(x)=\lambda(x) \delta_{i j} \delta_{k l}+\mu(x)\left(\delta_{i k} \delta_{j l}+\delta_{i l} \delta_{j k}\right) .
$$

Assume that $\lambda, \mu$ are locally Lipschitz in $\Omega$ and $\lambda+2 \mu>0, \mu>0$ for all $x \in \Omega$. Then (1.2) possesses the UCP.

\section{Applications to inVERSE problems}

As mentioned in the Introduction, we would like to investigate some applications of the UCP proved in the previous section to the object identification problem. Another application of the UCP to the limiting absorption principle for the same system was considered by the authors in [19]. To study our inverse problems here, the Runge approximation property with Dirichlet constraints for (1.2) is a key ingredient. It has been known that this Runge property is an easy consequence of the UCP. Its proof can be found, for example, in [11] or [12].

Theorem 3.1. Let $\mathcal{O}$ and $\Omega$ be two open bounded domains with Lipschitz boundaries such that $\overline{\mathcal{O}} \subset \Omega$. Assume that all components of $C$ are uniformly Lipschitz functions in $\Omega$. Moreover, suppose that the quadratic pencil $\Lambda_{11} p^{2}+\Lambda_{12} p+\Lambda_{22}$ has at least one eigenpair $(\theta(x), z(x))$ which is uniformly Lipschitz in $\Omega$ and $[z, \bar{z}]$ is nonsingular for all $x \in \bar{\Omega}$. Denote $\Gamma_{0}$ a subset of the boundary $\partial \Omega$. Let $u \in H^{1}(\mathcal{O})$ satisfy

$$
\mathcal{L}_{C} u=0 \text { in } \mathcal{O} .
$$

Then for any compact subset $K \subset \mathcal{O}$ such that $\Omega \backslash K$ is connected and any $\varepsilon>0$, there exists $w \in H^{1}(\Omega)$ satisfying

$$
\mathcal{L}_{C} w=0 \quad \text { in } \Omega
$$

with $\operatorname{supp}\left(\left.w\right|_{\partial \Omega}\right) \subset \Gamma_{0}$ such that

$$
\|w-u\|_{H^{1}(K)}<\varepsilon
$$

Remark. Under the assumptions of Theorem 3.1. we can extend $C$, denoted by $\hat{C}$, to a slightly larger domain $\hat{\Omega}$ such that the same assumptions are satisfied for $\hat{C}$ in $\hat{\Omega}$. Therefore, the UCP holds for (1.2) with $\hat{C}$ in $\hat{\Omega}$.

In this section we consider the inverse problem of identifying inclusions or cavities embedded in an anisotropic elastic plane region by boundary measurements. To begin, assume that $D$ is an open subset of $\Omega$ with Lipschitz boundary such that $\Omega \backslash \bar{D}$ is connected. The domain $D$ stands for the region of the inclusion or cavity embedded in $\Omega$. In the degenerate case where the domain $D$ represents the crack, the inverse problem of identifying $D$ by near-field measurements was consider in [16] and [17. To simply our presentation, we will not discuss this matter here. 
Let the reference elastic tensor $C(x)$ satisfy the conditions given in Theorem 3.1, Here we require that the elastic tensor $C(x)$ satisfies the strong convexity condition, namely, there exists $\kappa>0$ such that for any symmetric matrix $E$ we have

$$
C(x) E \cdot E \geq \kappa \sum_{i, j} E_{i j} E_{i j}=\kappa|E|^{2} \quad \forall x \in \Omega .
$$

It is obvious that (3.1) implies the strong ellipticity condition (2.1). Next we assume that $\tilde{C}(x)$ is some fourth-rank symmetric tensor with $L^{\infty}$ components such that $C+\chi_{D} \tilde{C}$ satisfies the strong convexity condition (3.1), where $\chi_{D}$ denotes the characteristic function of $D$. Then it is easy to show that there exists a unique solution $u \in H^{1}(\Omega)$ to

$$
\left\{\begin{array}{l}
\nabla \cdot\left(\left(C+\chi_{D} \tilde{C}\right) \nabla u\right)=0 \text { in } \Omega \\
u=f \quad \text { on } \partial \Omega
\end{array}\right.
$$

for any $f \in H^{1 / 2}(\partial \Omega)$. So we can define the Dirichlet-to-Neumann (displacementto-traction) map $\Lambda_{\text {inc }}: H^{1 / 2}(\partial \Omega) \rightarrow H^{-1 / 2}(\partial \Omega)$ by

$$
\Lambda_{\text {inc }}(f)=\left.(C \nabla u) \nu\right|_{\partial \Omega}
$$

where $\nu$ stands for the unit outer normal of $\partial \Omega$ (or $\partial D$ ). Equivalently, $\Lambda_{i n c}$ can be defined by the formula

$$
\left\langle\Lambda_{i n c}(f), g\right\rangle=\int_{\Omega}\left(C+\chi_{D} \tilde{C}\right) \nabla u \cdot \nabla v d x,
$$

where $v \in H^{1}(\Omega)$ with $\left.v\right|_{\partial \Omega}=g$. The region $D$ is called an inclusion if the medium in $D$ is different from the reference medium. To describe it more precisely, we assume that $\tilde{C}$ satisfies the following jump condition:

$\forall x \in \partial D, \exists C_{x}>0, \exists \delta_{x}>0$ such that $\tilde{C}(y) E \cdot E \geq C_{x}|E|^{2}$ or $\tilde{C}(y) E \cdot E \leq-C_{x}|E|^{2}$

for almost all $y \in B_{\delta_{x}}(x) \cap D$ and any symmetric matrix $E$. We are interested in the following inverse problem

IP.I Reconstruct the inclusion $D$ from the knowledge of $\left.\Lambda_{\text {inc }}(f)\right|_{\Gamma_{0}}$ for infinitely many $f \in H^{1 / 2}(\partial \Omega)$ with $\operatorname{supp}(f) \subset \Gamma_{0}$, where $\Gamma_{0}$ is a nonempty subset of $\partial \Omega$.

Likewise, in the extreme case, if the tensor $\tilde{C}$ becomes $-C$, then the domain $D$ corresponds to a cavity. In the same way, we can prove that there exists a unique solution $u \in H^{1}(\Omega \backslash \bar{D})$ to the following boundary value problem:

$$
\left\{\begin{array}{l}
\nabla \cdot(C \nabla u)=0 \quad \text { in } \Omega \backslash \bar{D}, \\
(C \nabla u) \nu=0 \quad \text { on } \partial D, \quad(C \nabla u) \nu=g \quad \text { on } \partial \Omega
\end{array}\right.
$$

for any $g \in H^{1 / 2}(\partial \Omega)$. Therefore, we can define the Dirichlet-to-Neumann map $\Lambda_{\text {cav }}: H^{1 / 2}(\partial \Omega) \rightarrow H^{-1 / 2}(\partial \Omega)$ by

$$
\Lambda_{\text {cav }}(g)=\left.(C \nabla u) \nu\right|_{\partial \Omega} .
$$

Similarly, we will consider the inverse problem

IP.C Reconstruct the cavity $D$ from the knowledge of $\left.\Lambda_{\text {cav }}(g)\right|_{\Gamma_{0}}$ for infinitely many $g$ with $\operatorname{supp}(g) \subset \Gamma_{0}$.

Note that uniqueness theorems of determining the inclusion or cavity embedded in an elastic body have been established in [11] and [12, where the reference medium is assumed to be either inhomogeneous isotropic or anisotropic with homogeneous 
or analytic elasticity tensors. Besides, a reconstruction algorithm for recovering the cavity is given in [12. A similar algorithm can be developed for the inclusion case.

Having the Runge approximation property Theorem 3.1 at hand, we can now apply the methods in [11] and [12] to solve IP.I and IP.C. For IP.I, we prove that (see [1])

Theorem 3.2 (Identification of inclusion). Let $\left(D_{1}, \tilde{C}_{1}\right)$ and $\left(D_{2}, \tilde{C}_{2}\right)$ be two sets of inclusion data satisfying all conditions stated in this section. If

$$
\Lambda_{i n c_{1}}(f)=\Lambda_{i n c_{2}}(f) \quad \text { on } \quad \Gamma_{0}
$$

for all $f \in H^{1 / 2}(\partial \Omega)$ with $\operatorname{supp}(f) \subset \Gamma_{0}$, then

$$
D_{1}=D_{2} \text {. }
$$

The proof of Theorem 3.2 is based on integral inequalities

$$
\int_{D}\left\{C^{-1}-(C+\tilde{C})^{-1}\right\} C \nabla w \cdot C \nabla w d x \leq\left\langle\left(\Lambda_{i n c}-\Lambda_{\emptyset}\right) f, f\right\rangle \leq \int_{D} \tilde{C} \nabla w \cdot \nabla w d x
$$

where $w \in H^{1}(\Omega)$ solves

$$
\left\{\begin{array}{l}
\nabla \cdot(C \nabla w)=0 \quad \text { in } \Omega, \\
\left.w\right|_{\partial \Omega}=f
\end{array}\right.
$$

and $\Lambda_{\emptyset}$ is the Dirichlet-to-Neumann map when $D$ is absent, namely, $\Lambda_{\emptyset}$ is the map associated with (3.4). Here $C^{-1}$ (also $\left.(C+\tilde{C})^{-1}\right)$ is called the compliance tensor (see, e.g., 6]). Note that we do not assume $\tilde{C}_{1}=\tilde{C}_{2}$ in Theorem 3.2 . Also, the regularity of the medium inside of the inclusions is only assumed to be essentially bounded. Theorem 3.2 provides the uniqueness result in determining the inclusion embedded in an inhomogeneous anisotropic elastic plane region by the localized Dirichlet to Neumann map. For the sake of completeness, we want to briefly describe a reconstruction algorithm for identifying the inclusion. Let $y \in \Omega$ and $G_{0}(\cdot ; y)$ be the fundamental solution for the operator $\nabla \cdot C(y) \nabla$. One can find $e(\cdot ; y)$ such that

$$
\nabla \cdot(C(x) \nabla e(\cdot ; y))=0 \quad \text { in } \quad \Omega \backslash\{y\}
$$

and

$$
\left(e(\cdot ; y)-G_{0}(\cdot-y ; y) b\right)_{y \in \Omega} \quad \text { is bounded in } H^{1}(\Omega),
$$

where $b$ is a nonzero constant vector. The proof for the existence of $e(\cdot ; y)$ is elementary and therefore we leave it to the reader. It has been proved in [1] that if $y \in \partial D$, then

$$
\int_{D \cap B_{r}(y)}\left|\operatorname{Sym} \nabla\left\{G_{0}(x-y ; y) b\right\}\right|^{2} d x=\infty
$$

for any ball $B_{r}(y)$ centered at $y$ with radius $r$ and nonzero vector $b$, where $\operatorname{Sym}(\cdot)$ denotes the symmetric part of a matrix.

A continuous map $c:[0,1] \rightarrow \bar{\Omega}$ is called a needle if it satisfies (i) $c(0), c(1) \in \partial \Omega$; (ii) $c(t) \in \Omega$ for $0<t<1$. In view of Theorem 3.1, we can see that for each needle and $t \in(0,1)$, there exists a sequence $\left\{f_{j}\right\}=\left\{f_{j}(\cdot ; c(t))\right\}$ in $H^{1 / 2}(\partial \Omega)$ with $\operatorname{supp}\left(f_{j}\right) \subset \Gamma_{0}$ such that the solution $w_{j}$ of (3.4) with $f=f_{j}$ satisfies $w_{j} \rightarrow e(\cdot ; c(t))$ in $H_{\text {loc }}^{1}\left(\Omega \backslash\left\{c\left(t^{\prime}\right): 0<t^{\prime} \leq t\right\}\right)$ as $j \rightarrow \infty$. We call $\left\{f_{j}\right\}$ a fundamental sequence with respect to $\Gamma_{0}$. For each needle $c$, define

$$
t(c)=\sup \{0<s<1: C(t) \in \Omega \backslash \bar{D}(0<t<s)\} .
$$


It should be noted that $0<t(c) \leq 1$, and if $t(c)=1$, then $c$ never touches $\partial D$. On the other hand, if $t(c)<1$, then $c$ touches $\partial D$ at $t=t(c)$ at the first time. Since $\Omega \backslash \bar{D}$ is connected, we have that

$$
\partial D=\{c(t(c)): c \text { is a needle and } t(c)<1\} .
$$

Denote

$$
\mathcal{I}_{i n c}(t, c)=\lim _{j \rightarrow \infty}\left\langle\left(\Lambda_{i n c}-\Lambda_{\emptyset}\right) f_{j}(\cdot ; c(t)), f_{j}(\cdot ; c(t))\right\rangle
$$

and

$$
\mathcal{I}_{\text {inc }}(c)=\left\{0<s<1: \mathcal{I}_{\text {inc }} \text { exists for all } 0<t<s \text { and } \sup _{0<t<s}\left|\mathcal{I}_{\text {inc }}(t, c)\right|<\infty\right\} .
$$

Using (3.2), (3.3), (3.5) and pursuing the arguments in [11, we can show that $\mathcal{T}_{\text {inc }}(c)=(0, t(c))$ and therefore $t(c)=\sup \mathcal{T}_{\text {inc }}(c)$ (see similar arguments in [12]). In summary, we have a reconstruction algorithm for determining the inclusion as follows.

\section{Reconstruction algorithm for IP.I.}

(i) For each needle $c$ and each $t \in(0,1)$, find the fundamental sequence $\left\{f_{j}(\cdot ; c(t))\right\}$ with respect to $\Gamma_{0}$.

(ii) Compute $\mathcal{T}_{\text {inc }}(c)$ and set $t(c)=\sup \mathcal{T}_{\text {inc }}(c)$.

(iii) Use the formula (3.6) to reconstruct $\partial D$.

Now for IP.C, we show that (see [12])

Theorem 3.3 (Identification of cavity). Assume that $D_{1}$ and $D_{2}$ are two cavities and $\Omega \backslash \bar{D}_{1}$ and $\Omega \backslash \bar{D}_{2}$ are connected. Let

$$
\Lambda_{\text {cav }_{1}}(f)=\Lambda_{\text {cav }_{2}}(f) \quad \text { on } \quad \Gamma_{0}
$$

for all $f \in H^{1 / 2}(\partial \Omega)$ with $\operatorname{supp}(f) \subset \Gamma_{0}$; then $D_{1}=D_{2}$.

As for reconstructing the cavity, we follow the lines of the above algorithm and define

and

$$
\mathcal{I}_{\text {cav }}(t, c)=\lim _{j \rightarrow \infty}\left\langle\left(\Lambda_{\emptyset}-\Lambda_{\text {cav }}\right) f_{j}(\cdot ; c(t)), f_{j}(\cdot ; c(t))\right\rangle
$$

$$
\mathcal{T}_{\text {cav }}(c)=\left\{0<s<1: \mathcal{I}_{\text {cav }} \text { exists for all } 0<t<s \text { and } \sup _{0<t<s} \mathcal{I}_{\text {cav }}(t, c)<\infty\right\} .
$$

Note that $\left\langle\left(\Lambda_{\emptyset}-\Lambda_{\text {cav }}\right) f, f\right\rangle \geq 0$ for all $f \in H^{1 / 2}(\partial \Omega)$. Now using (3.5) and the inequalities

$$
\frac{1}{M} \int_{D}|\nabla e(x ; c(t))|^{2} d x \leq \mathcal{I}_{\text {cav }}(t, c) \leq M \int_{D}|\nabla e(x ; c(t))|^{2} d x
$$

for some constant $M>0$, one can prove that $\mathcal{T}_{\text {cav }}(c)=(0, t(c))$ and thus $t(c)=$ $\sup \mathcal{T}_{\text {cav }}(c)$ (see the arguments in [12]). So a reconstruction algorithm for identifying the cavity is described as follows.

\section{Reconstruction algorithm for IP.C.}

(i) For each needle $c$ and each $t \in(0,1)$, find the fundamental sequence $\left\{f_{j}(\cdot ; c(t))\right\}$ with respect to $\Gamma_{0}$.

(ii) Compute $\mathcal{T}_{\text {cav }}(c)$ and set $t(c)=\sup \mathcal{T}_{\text {cav }}(c)$.

(iii) Use the formula (3.6) to reconstruct $\partial D$. 


\section{REFERENCES}

[1] D.D. Ang, M. Ikehata, D.D. Trong and M. Yamamoto, Unique continuation for a stationary isotropic Lamé system with varaiable coefficients, Comm. in PDE, 23 (1998), 371-385. MR.1608540 (98j:35049)

[2] G. Alessandrini and A. Morassi, Strong unique continuation for the Lamé system of elasticity, Comm. in PDE., 26 (2001), 1787-1810. MR1865945 (2003j:35030)

[3] T. Carleman, Sur un problèm d'unicité pour les systemes d'équations aux dérivées partielles à deux varaibles indépendentes, Ark. Mat., Astr. Fys., 26B (1939), 1-9.

[4] B. Dehman and L. Rabbiano, La propriété du prolongement unique pour un système elliptique: le système de Lamé, J. Math. Pures Appl., 72 (1993), 475-492. MR1239100 (94h:35051)

[5] A. Douglis, Uniqueness in Cauchy problem for elliptic systems of equations, Comm. Pure Appl. Math., 6 (1953), 291-298. MR0064278 (16:257d)

[6] M. Gurtin, The Linear Theory of Elasticity, Mechanics of Solids, Vol. II (Thruesdell C., ed.), Springer-Verlag, Berlin, 1984.

[7] T. Hildebrandt and L. Graves, Implicit functions and their differentials in general analysis, Trans. Amer. Math. Soc., 29 (1927), 127-153. MR1501380

[8] G. Hile and M. Protter, Unique continuation and the Cauchy problem for first order systems of partial differential equations, Comm. in PDE, 1 (1976), 437-465. MR0481439 (58:1555)

[9] M. Ikehata, Reconstruction of the shape of the inclusion by boundary measurements, Comm. in PDE, 23 (1998), 1459-1474. MR1642619 (99f:35222)

[10] M. Ikehata, The probe method and its applications, Inverse problems and related topics (Kobe, 1998), 57-68, Chapman and Hall/CRC Res. Notes Math., 419, Chapman \& Hall/CRC, Boca Raton, FL, 2000. MR 1761338(2001c:35245)

[11] M. Ikehata, G. Nakamura, and K. Tanuma, Identification of the shape of the inclusion in the anisotropic elastic body, Appl. Anal., 72 (1999), 17-26. MR.1775432 (2001d:74026)

[12] M. Ikehata and G. Nakamura, Reconstruction of cavity from boundary measurements, preprint.

[13] V. Isakov, On uniqueness of recovery of a discontinuous conductivity coefficients, Comm. Pure Appl. Math, 41 (1988), 865-877. MR0951742 (90f:35205)

[14] C.-L. Lin, Strong unique continuation for an elasticity system with residual stress, Indiana Univ. Math. J., 53 (2004), 557-582. MR2060045 (2005f:35033)

[15] C.-L. Lin and J.-N. Wang, Strong unique continuation for the Lamé system with Lipschitz coefficients, Math. Annalen, to appear.

[16] G. Nakamura, G. Uhlmann, and J.-N. Wang, Reconstruction of cracks in an anisotropic elastic medium, J. Math. Pures Appl., 82 (2003), 1251-1276. MR2020922 (2005b:35299)

[17] G. Nakamura, G. Uhlmann, and J.-N. Wang, Reconstruction of cracks in an inhomogeneous anisotropic medium using point sources, Advances in Appl. Math., to appear.

[18] G. Nakamura and J.-N. Wang, Unique continuation for an elasticity system with residual stress and its applications, SIAM J. Math. Anal., 35 (2003), 304-317. MR2001103 (2004k:35037)

[19] G. Nakamura and J.-N. Wang, The limiting absorption principle for the two-dimensional inhomogeneous anisotropic elasticity system, Trans. Amer. Math. Soc., 358 (2006), 147-165.

[20] G. Uhlmann, Developments in inverse problems since Calderon's foundational paper, Harmonic analysis and partial differential equations (Chicago, IL, 1996), 295-345, Chicago Lectures in Math., Univ. Chicago Press, Chicago, IL, 1999. MR1743870 (2000m:35181)

[21] N. Weck, Unique continuation for systems with Lamé principal part, Math. Methods Appl. Sci., 24 (2001), 595-605. MR1834916 (2002f:35048)

[22] E. Zeidler, Nonlinear Function Analysis and its Applications, I. Fixed-Point Theorems, Springer-Verlag, New York, 1986. MR0816732 (87f:47083)

Department of Mathematics, Hokkaido University, Sapporo 060-0810, Japan

E-mail address: gnaka@math.sci.hokudai.ac.jp

Department of Mathematics, National Taiwan University, Taipei 106, Taiwan

E-mail address: jnwang@math.ntu.edu.tw 